\title{
Towards a new image processing system at Wendelstein 7-X: From spatial calibration to characterization of thermal events
}

Cite as: Rev. Sci. Instrum. 89, 123503 (2018); https://doi.org/10.1063/1.5045560

Submitted: 21 June 2018 . Accepted: 25 November 2018 . Published Online: 13 December 2018

F. Pisano (D), B. Cannas, M. W. Jakubowski (D), H. Niemann, A. Puig Sitjes, G. A. Wurden (D), and W7-X Team

\section{ARTICLES YOU MAY BE INTERESTED IN}

Infrared imaging systems for wall protection in the W7-X stellarator (invited)

Review of Scientific Instruments 89, 10E116 (2018); https://doi.org/10.1063/1.5038634

Optically isolated millimeter-wave detector for the Toroidal Plasma Experiment

Review of Scientific Instruments 89, 124702 (2018); https://doi.org/10.1063/1.5053991

Contributed Review: A review of compact interferometers

Review of Scientific Instruments 89, 121501 (2018); https://doi.org/10.1063/1.5052042

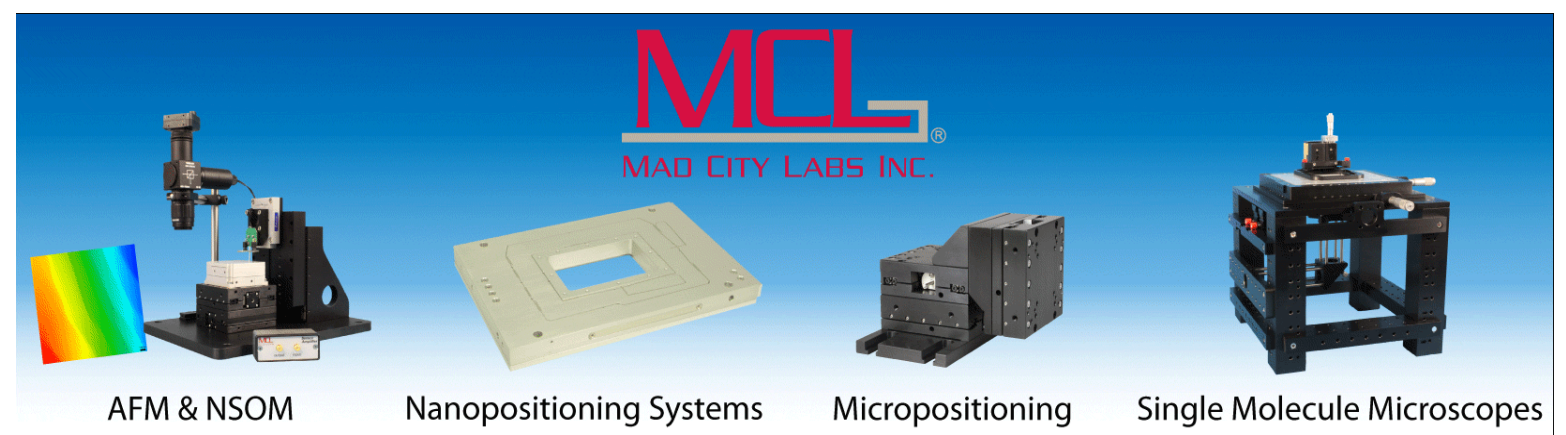




\title{
Towards a new image processing system at Wendelstein 7-X: From spatial calibration to characterization of thermal events
}

\author{
F. Pisano, ${ }^{1}$ B. Cannas, ${ }^{1}$ M. W. Jakubowski, ${ }^{2,3}$ H. Niemann, ${ }^{2}$ A. Puig Sitjes, ${ }^{2}$ G. A. Wurden, ${ }^{4}$ \\ and W7-X Team ${ }^{2, a)}$ \\ ${ }^{1}$ Department of Electrical and Electronic Engineering, University of Cagliari, Via Marengo 2, \\ Cagliari 09123, Italy \\ ${ }^{2}$ Max-Planck-Institut für Plasmaphysik, Teilinstitut Greifswald, Wendelsteinstraße 1, \\ Greifswald D-17491, Germany \\ ${ }^{3}$ University of Szczecin, Institute of Physics, Szczecin 70-451, Poland \\ ${ }^{4}$ Los Alamos National Laboratory, Los Alamos, New Mexico 87545, USA
}

(Received 21 June 2018; accepted 25 November 2018; published online 13 December 2018)

\begin{abstract}
Wendelstein 7-X (W7-X) is the most advanced fusion experiment in the stellarator line and is aimed at proving that the stellarator concept is suitable for a fusion reactor. One of the most important issues for fusion reactors is the monitoring of plasma facing components when exposed to very high heat loads, through the use of visible and infrared (IR) cameras. In this paper, a new image processing system for the analysis of the strike lines on the inboard limiters from the first W7-X experimental campaign is presented. This system builds a model of the IR cameras through the use of spatial calibration techniques, helping to characterize the strike lines by using the information given by real spatial coordinates of each pixel. The characterization of the strike lines is made in terms of position, size, and shape, after projecting the camera image in a 2D grid which tries to preserve the curvilinear surface distances between points. The description of the strike-line shape is made by means of the Fourier Descriptors. https://doi.org/10.1063/1.5045560
\end{abstract}

\section{INTRODUCTION}

Wendelstein 7-X (W7-X) is a helical axis optimized superconducting stellarator, in operation since 2015 , investigating one of the potential concepts of plasma magnetic confinement in nuclear fusion reactors. In this context, the processing in a controlled way of the large portion of energy created inside the reactor is one of the most important issues.

In $\mathrm{W} 7-\mathrm{X}$, where the plasma is confined by a special 3D shaped magnetic field, the concept of island divertor is investigated. The divertor is the main plasma facing component (PFC) intersected by the magnetic field lines. During the steady state operation of W7-X, it will be an actively water-cooled CFC (Carbon Fiber Composite) based unit, with a surface area of approximately $19 \mathrm{~m}^{2}$, designed to withstand heat loads of $10 \mathrm{MW} / \mathrm{m}^{2}$ locally in steady-state operation. A very important prerequisite for safe operation of a steady-state device is to monitor the materials when exposed to very high heat loads. In case local loads exceed the permitted limit, there is a risk of delamination, i.e., creation of defects in the PFC surface, and failure of the components.

Infrared thermography is an essential tool in measuring the surface temperature and estimating the heat loads in the present-day fusion devices. ${ }^{1}$ It is based on the conversion into temperature, according to Planck's law, of the light emitted by the observed surface, typically in the wavelength ranges of 3-5 $\mu \mathrm{m}$ or 8-14 $\mu \mathrm{m}$. A typical instrument, used to make such measurements, consists of an infrared camera and a set of optics, which image the observed plasma facing components onto the camera sensor. The taken images need to be

a) See the authors list in R. C. Wolf et al., Nucl. Fusion 57, 102020 (2017). compared with the projection in the image plane of the PFC CAD (Computer-Aided Design) models in order to better understand observed features: e.g., thermal events occurring in areas where high heat loads are expected should be treated separately than those occurring on other PFCs. Thus, as the discharges planned for $\mathrm{W} 7-\mathrm{X}$ will have a length of up to $30 \mathrm{~min}$, a huge amount of data will be created and a fully automatic analysis of the infrared images is required.

As the in-vessel components of the machine can be critically damaged, a real time system response based on the evaluation of the infrared (IR) measurements is already under development. This system will be capable of providing a rapid signal to the main control system of W7-X in order to protect the machine. ${ }^{2}$ As an implication, the real-time image processing will be challenging and heavy in terms of processing time and computational complexity. For this reason, a computeraided analysis of images is a key point to develop tools able to efficiently and automatically process, detect, and analyze this information.

Plasma operation of W7-X follows a staged approach in accordance with the successive completion of the in-vessel components. During steady state operation, planned for early 2020s, W7-X will be equipped with a water-cooled island divertor and a water-cooled graphite armor for the endangered parts of the first wall. During the first operational phase (OP1.1), no divertors were present and the plasma was limited to an effective minor radius of $a=0.49 \mathrm{~m}$ by five inboard uncooled graphite limiters. ${ }^{3}$ To avoid plasma contact with the metallic walls, a magnetic field configuration was developed, having a rotational transform at the plasma edge above 5/6, with a 5/6 island chain lying just inside the plasma volume. In this way, the plasma edge comes into contact with the limiter 
surface, creating a heat load pattern commonly called "strikeline." The magnetic configuration, in a poloidal cut through the device, is shown in Fig. 1. As can be seen, the edge magnetic field lines cross the limiter surface, where the strike-line should be positioned. In this paper, we present a new method to aid the infrared image analysis at W7-X with adapting the plasma vessel models, tested on OP1.1 data with the five in-board graphite limiters.

This paper is organized as follows: In Sec. II, an overview of the IR system for the observation of the limiters, an introduction on thermal events, in particular strike-lines, and objectives of the image processing, are given. Section III describes the spatial calibration process. In Sec. IV, two mapping problems are faced: the first mapping is done by projecting the camera

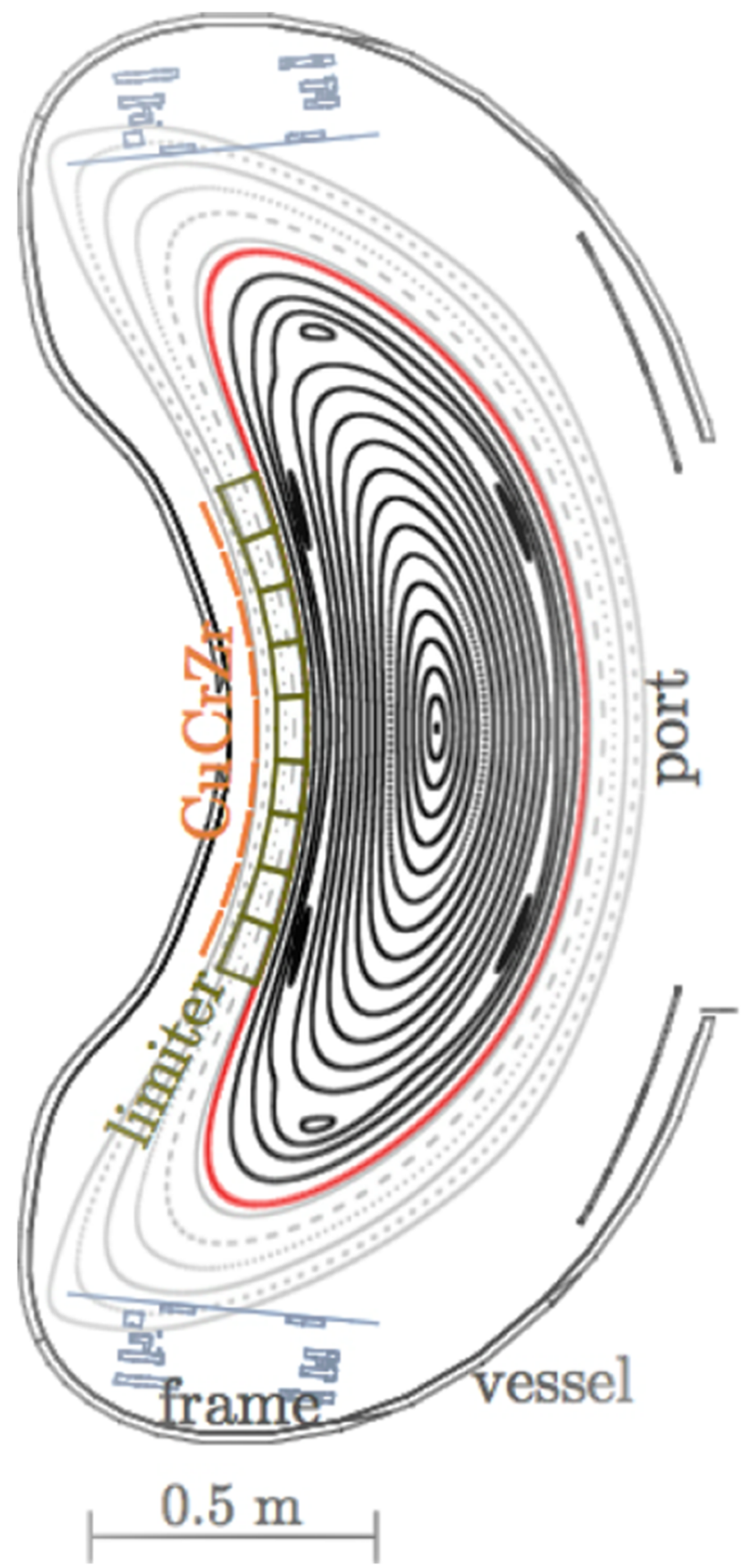

FIG. 1. Magnetic flux surface calculated for the limiter configuration of Wendelstein 7-X during its initial campaign. images onto the CAD surface; the second mapping is done by projecting the $3 \mathrm{D}$ surface on a $2 \mathrm{D}$ plane, trying to preserve distances between points. In Sec. V, the strike-line characterization process is explained. Finally, in Sec. VI, the conclusions and the future work perspectives are given.

\section{IR CAMERA SYSTEM AT W7-X}

From early 2020s, ten actively cooled divertor units, designed to withstand $10 \mathrm{MW} / \mathrm{m}^{2}$ in steady state, will be used for power and particle exhaust. A set of ten infrared and visible endoscopes will monitor the divertors and baffle surfaces. This monitoring system must identify areas with too high temperature, distinguish them from other events, and send alarms. Therefore, accurate and fast image analysis of infrared camera data is essential for W7-X operation. Following the staged approach of W7-X completion, during the first campaign, a basic IR system for the observation of the graphite limiters has been installed. ${ }^{4}$ This system consisted of three types of cameras: near-infrared (NIR) cameras, one long-wavelengthinfrared (LWIR) system, and one mid-wavelength-infrared (MWIR) system.

The NIR systems consisted of ten immersion tubes, measuring at $0.9 \mu \mathrm{m}$. The LWIR system consisted of a modified micro-bolometer camera, measuring at $8-14 \mu \mathrm{m}, 50 \mathrm{~Hz}, 640 \times$ 480 pixels, viewing the left side of the limiter in module 5 with a spatial resolution of ca. $5 \mathrm{~mm} /$ pixel $^{5-8}$ The MWIR system consisted of a high-resolution IR camera, measuring at 3-5 $\mu \mathrm{m}$, $125 \mathrm{~Hz}$ full-frame rate, $1344 \times 768$ pixels, viewing the central tiles of the limiter in module 3 with a spatial resolution of ca. $1 \mathrm{~mm} /$ pixel. $^{9,10}$ The LWIR and MWIR systems have the capability of observing, respectively, the full left strike-line in the limiter of module 5 and part of the two strike-lines in just five tiles of the limiter in module 3 . On the contrary, NIR cameras suffered from plasma emission near the limiter surface and delivered no useful data.

Figure 2 shows the position and the view cone (towards the limiters) of the MWIR and LWIR camera systems onto a simplified 3D CAD model of W7-X (.stl prototype). The limiters are highlighted in light blue, and the cameras in violet. The photograph of the limiter in module 5 is also presented in Fig. 2(d), where the fields of views of the two infrared cameras are shown. Note that the forward-looking infrared (FLIR) camera actually observes the limiter in module 3 .

In the initial W7-X campaign, the IR system was used with the aim of assessing the power balance and the heat load distribution over the inboard limiters. The main feature in the heat load pattern on the limiter surface is commonly called "strike-line," and it is created by a direct contact of plasma with the limiter surface. In the case of W7-X, its elongated shape is defined by the magnetic topology of field lines and plasma transport at the edge. ${ }^{10}$

From the two camera systems, LWIR and MWIR, it is possible to see two contact strike-lines on the limiter surface, ${ }^{8,10}$ which became the dominant infrared structure. As can be noticed in Fig. 3, the LWIR system reveals only the left strike-line in the limiter in module 5, whereas MWIR only displays the central part of the two strike-lines in the limiter in module 3 . 
a)

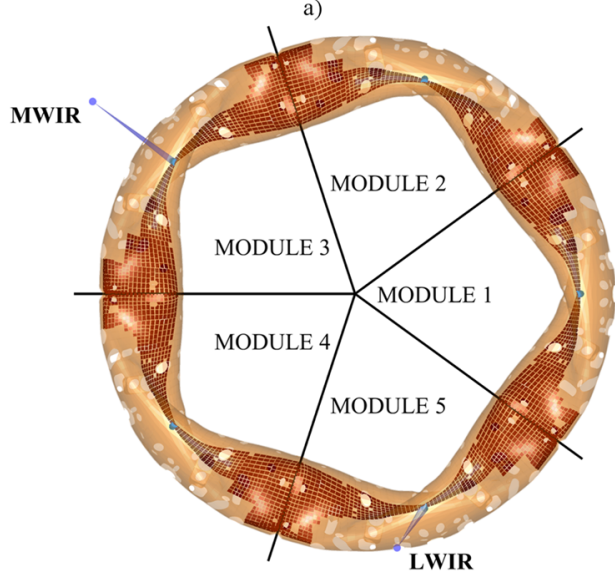

b)

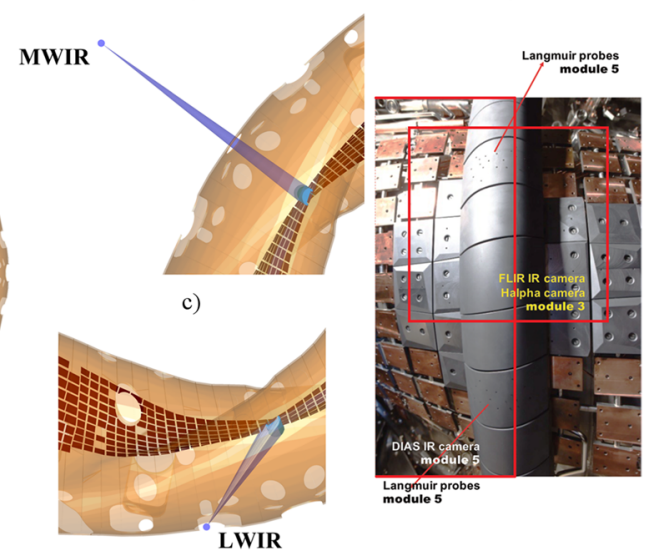

FIG. 2. (a) Position and views on the limiters of the MWIR and LWIR camera systems, (b) zoomed-in MWIR camera view, (c) zoomed-in LWIR camera view, and (d) the graphite limiter on the inboard side of Wendelstein 7-X in module 5; pins of Langmuir probes are visible above and below the midplane; the red rectangles indicate the limiter camera views, superimposed on the limiter of module 5 (DIAS and FLIR denote LWIR and MWIR cameras, respectively). a)

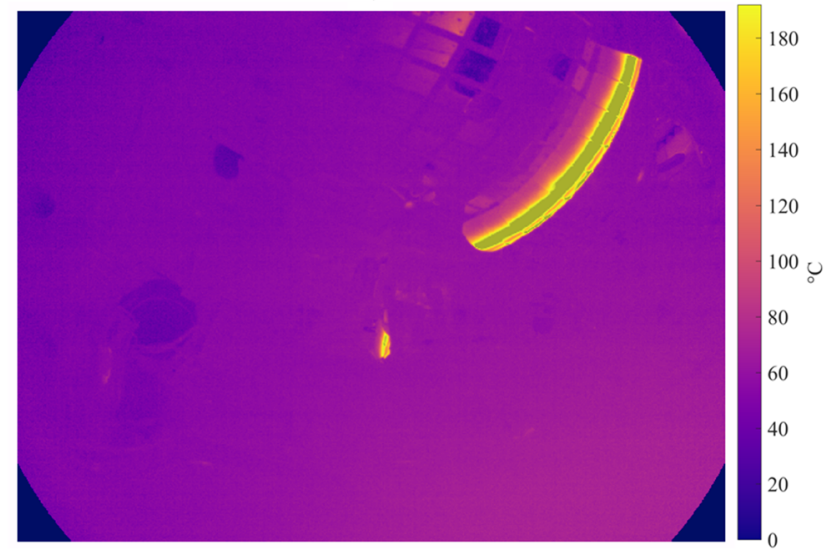

b)

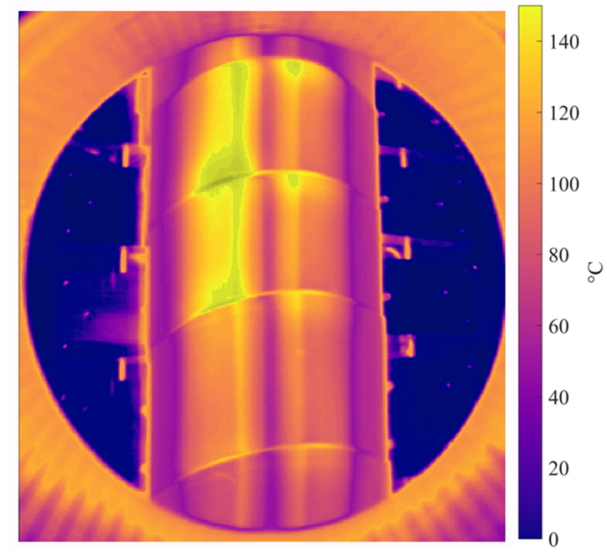

FIG. 3. (a) LWIR camera system view and (b) MWIR camera system view.

\section{SPATIAL CALIBRATION}

In order to find a one-to-one map between the points of the limiter surface and the pixels in the image, a spatial calibration phase is necessary. Spatial camera calibration includes finding the extrinsic parameters, the intrinsic parameter matrix, and the lens distortion coefficients, which are able to reproduce the correct mapping between 3D CAD points and the image points. The extrinsic parameters represent the effective location and orientation of the equivalent pinhole camera in the 3D world, whereas the intrinsic parameters represent the position of the principal point in the image plane and the focal length of the camera in pixels. Normally there are two different types of lens distortion components, radial and tangential, with respect to the ideal image plane, but in our case, the tangential component can be neglected. Finding a correct set of parameters is especially important for wide angle lenses, as they lead to large image distortions.

Each camera and its view have been modeled by using the usual pinhole model, ${ }^{11,12}$ which models the relationship between the $i$ th $3 \mathrm{D}$ point $\boldsymbol{w}_{i}=\left[X_{i} Y_{i} Z_{i}\right]$ inside the camera field of view and the corresponding image point $\boldsymbol{p}_{i}$ in pixel coordinates. The origin of the $3 \mathrm{D}$ coordinate system $\left(X_{i} Y_{i} Z_{i}\right)$ is the center of the stellarator.

The image point is described through homogeneous coordinates $\boldsymbol{p}_{i}=\left[\begin{array}{lll}x_{i} & y_{i} & 1\end{array}\right]$. Indeed, by adding a "fictitious" third coordinate, translations and rotations can be expressed as matrices multiplications. ${ }^{11,12}$ The image point is given by

$$
\left\{\begin{array}{l}
\boldsymbol{p}_{i}=\boldsymbol{c}_{i} \boldsymbol{K}_{r a d} \boldsymbol{K}, \\
d_{i} \boldsymbol{c}_{i}=\boldsymbol{w}_{i} \boldsymbol{R}+\boldsymbol{t}, \\
\boldsymbol{K}_{\text {rad }}=\operatorname{diag}\left(k_{r a d}, k_{r a d}, 1\right), \\
k_{\text {rad }}=1+k_{r 1} \rho_{i}^{2}+k_{r 2} \rho_{i}^{4}+k_{r 3} \rho_{i}^{6},
\end{array}\right.
$$

where $\boldsymbol{c}_{i}=\left[\alpha_{i} \beta_{i} 1\right]$ are the homogeneous coordinates in the camera reference system; $\boldsymbol{K}$ is the intrinsic parameter matrix; $k_{r 1}, k_{r 2}, k_{r 3}$ are the radial distortion coefficients defining the radial distortion matrix $\boldsymbol{K}_{\text {rad }}, \rho_{i}=\sqrt{\alpha_{i}^{2}+\beta_{i}^{2}} ; d_{i}$ is a scale factor indicating the distance of the point from the camera eye in the direction of the optical axis of the camera; the rotation matrix $\boldsymbol{R}$ and the translation vector $\boldsymbol{t}$ define the extrinsic parameters.

A quite accurate estimation of the intrinsic camera parameters and the distortion parameters can be obtained with multiple views of a checkerboard-like object. ${ }^{13}$ Checkerboards are often used during camera calibration because they are simple to construct and their planar grid structure defines many natural interest points in an image. This method consists of three steps: at first, a raw estimation of the intrinsic parameter matrix $\boldsymbol{K}$ is made analytically; second, this estimation is refined by a nonlinear optimization technique based on the 


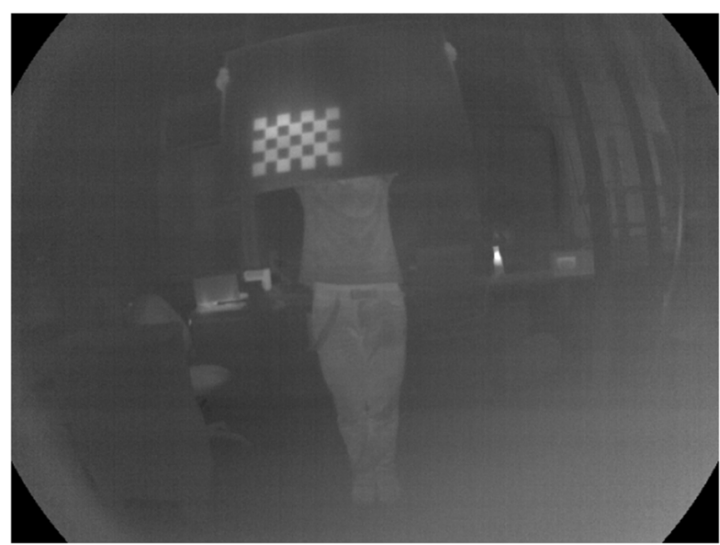

FIG. 4. Example of checkerboard measurement performed in the laboratory.

maximum likelihood criterion; finally, lens radial distortion is taken into account and the distortion parameters are first estimated analytically and then refined by a nonlinear optimization technique based on the maximum likelihood criterion. A set of multiple views of a checkerboard has been used to estimate the intrinsic parameter matrix and to correct the strong radial lens distortion for the LWIR camera system. In order to obtain the set of checkerboard images, the magnets of the checkerboard were heated up by a heat gun. An example of checkerboard view is shown in Fig. 4.

After acquiring multiple images with different positions of the checkerboard, a set of calibration points, for which both 3D and pixel coordinates were known, has been chosen. Figure 5(a) shows the calibration points (in red) used for spatial calibration of the LWIR camera system.

The camera coordinates $\alpha_{i}$ and $\beta_{i}$ of the $n$ calibration points can be evaluated by knowing the radial distortion coefficients and the intrinsic matrix calculated in the previous step, solving the equations in system (1). Then, a first estimation of the extrinsic parameters has been obtained by solving the linear system

$$
\left\{\begin{array} { c } 
{ d _ { i } \alpha _ { i } = \boldsymbol { w } _ { i } \boldsymbol { r } _ { 1 } + t _ { 1 } } \\
{ d _ { i } \beta _ { i } = \boldsymbol { w } _ { i } \boldsymbol { r } _ { 2 } + t _ { 2 } } \\
{ d _ { i } = \boldsymbol { w } _ { i } \boldsymbol { r } _ { 3 } + t _ { 3 } }
\end{array} \Rightarrow \left\{\begin{array}{l}
\left(\boldsymbol{w}_{i} \boldsymbol{r}_{3}+t_{3}\right) \alpha_{i}=\boldsymbol{w}_{i} \boldsymbol{r}_{1}+t_{1} \\
\left(\boldsymbol{w}_{i} \boldsymbol{r}_{3}+t_{3}\right) \beta_{i}=\boldsymbol{w}_{i} \boldsymbol{r}_{2}+t_{2}
\end{array}{ }^{2}=1, \ldots, n\right.\right.
$$

in the least-squares sense, where $\boldsymbol{r}_{1}, \boldsymbol{r}_{2}, \boldsymbol{r}_{3}$ are the columns of rotation matrix $\boldsymbol{R}$, and by considering the orthogonality of $\boldsymbol{R}$ as a nonlinear constraint, i.e., $\left\|\boldsymbol{r}_{1}\right\|=\left\|\boldsymbol{r}_{2}\right\|=\left\|\boldsymbol{r}_{3}\right\|=1$ and
$\boldsymbol{r}_{1}^{T} \boldsymbol{r}_{2}=\boldsymbol{r}_{2}^{T} \boldsymbol{r}_{3}=\boldsymbol{r}_{3}^{T} \boldsymbol{r}_{1}=0$. The solution has then been improved by minimizing the nonlinear function

$$
\frac{1}{n} \sum_{i=1}^{n}\left[\left(\alpha_{i}-\frac{\boldsymbol{w}_{i} \boldsymbol{r}_{1}+t_{1}}{\boldsymbol{w}_{i} \boldsymbol{r}_{3}+t_{3}}\right)^{2}+\left(\beta_{i}-\frac{\boldsymbol{w}_{i} \boldsymbol{r}_{2}+t_{2}}{\boldsymbol{w}_{i} \boldsymbol{r}_{3}+t_{3}}\right)^{2}\right]
$$

with the same nonlinear constraints on $\boldsymbol{R}$.

For the MWIR camera system, no checkerboard measurements are needed because of the really low lens distortion and the front position of the camera with respect to the limiter surface. Thus, a first estimation of extrinsic and intrinsic matrices has been made from the calibration points without taking care of distortion [see Fig. 5(b)], by solving the system

$$
\left\{\begin{array}{l}
d_{i} x_{i}=\left(\boldsymbol{w}_{i} \boldsymbol{R} \boldsymbol{k}_{3}+\boldsymbol{t} \boldsymbol{k}_{3}\right) x_{i}=\boldsymbol{w}_{i} \boldsymbol{R} \boldsymbol{k}_{1}+\boldsymbol{t}_{1} \\
d_{i} y_{i}=\left(\boldsymbol{w}_{i} \boldsymbol{R} \boldsymbol{k}_{3}+\boldsymbol{t} \boldsymbol{k}_{3}\right) y_{i}=\boldsymbol{w}_{i} \boldsymbol{R} \boldsymbol{k}_{2}+\boldsymbol{t k}_{2}
\end{array} \quad i=1, \ldots, n\right.
$$

in the least-squares sense, by imposing the nonlinear constraint $\left\|\boldsymbol{R}_{3}\right\|=\left\|\boldsymbol{k}_{3}\right\|=1$, where $\boldsymbol{k}_{1}, \boldsymbol{k}_{2}, \boldsymbol{k}_{3}$ are the columns of intrinsic matrix $\boldsymbol{K}$. The solution has been then improved by minimizing the nonlinear function

$$
\frac{1}{n} \sum_{i=1}^{n}\left[\left(x_{i}-\frac{\boldsymbol{w}_{i} \boldsymbol{R} \boldsymbol{k}_{1}+\boldsymbol{t} \boldsymbol{k}_{1}}{\boldsymbol{w}_{i} \boldsymbol{R} \boldsymbol{k}_{3}+\boldsymbol{t} \boldsymbol{k}_{3}}\right)^{2}+\left(y_{i}-\frac{\boldsymbol{w}_{i} \boldsymbol{R} \boldsymbol{k}_{2}+\boldsymbol{t} \boldsymbol{k}_{2}}{\boldsymbol{w}_{i} \boldsymbol{R} \boldsymbol{k}_{3}+\boldsymbol{t} \boldsymbol{k}_{3}}\right)^{2}\right]
$$

with the same nonlinear constraints. The model has been finally enhanced by adding a light distortion component, and minimizing the nonlinear function

$$
\frac{1}{n} \sum_{i=1}^{n}\left[\left(x_{i}-f_{x}\left(\boldsymbol{w}_{i}, \boldsymbol{R}, \boldsymbol{K}, \boldsymbol{k}_{r a d}\right)\right)^{2}+\left(y_{i}-f_{y}\left(\boldsymbol{w}_{i}, \boldsymbol{R}, \boldsymbol{K}, \boldsymbol{k}_{r a d}\right)\right)^{2}\right],
$$

where $f_{x}$ und $f_{y}$ are the projection functions from 3D coordinates to image coordinates, obtained by solving system (1) with the same nonlinear constraints.

\section{MAPPING OF THE CAMERA IMAGE TO CAD SURFACE AND 2D PLANE}

\section{A. Camera image to CAD surface mapping}

By knowing the camera model and the calibration parameters, it is possible to project the $i$ th vertex $\boldsymbol{w}_{i}=\left[X_{i} Y_{i} Z_{i}\right]$ of the CAD model onto the image plane and find its homogeneous coordinates $\boldsymbol{p}_{i}=\left[\begin{array}{lll}x_{i} & y_{i} & 1\end{array}\right]$.

The inverse procedure, i.e., finding the point in the CAD model related to each pixel in the image, needs more efforts. In fact, each pixel relates to one line of sight from the camera pupil, which consists of an infinite number of points. Given a)

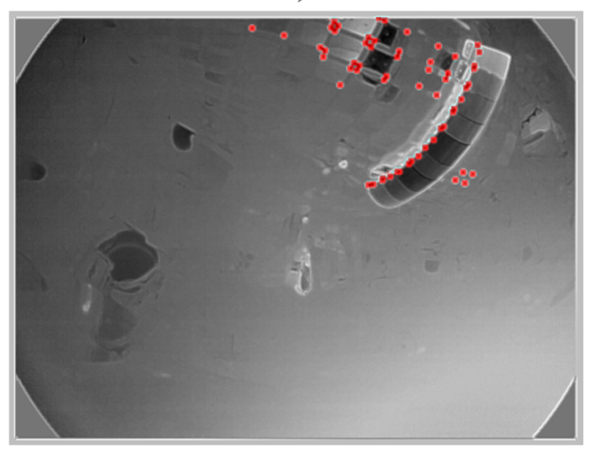

b)

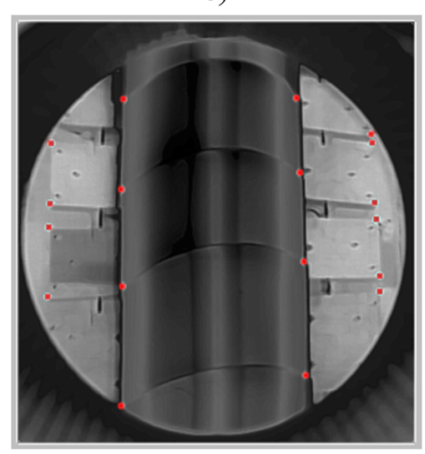

FIG. 5. Calibration points: (a) LWIR and (b) MWIR. 
a)

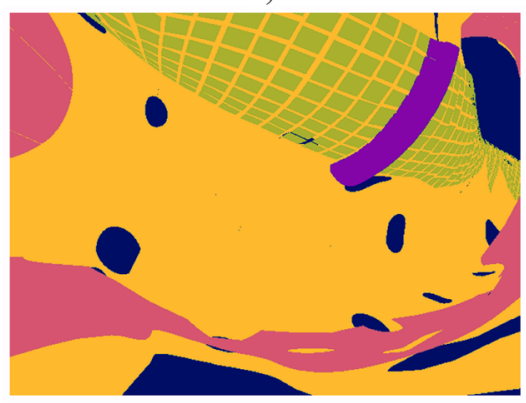

b)

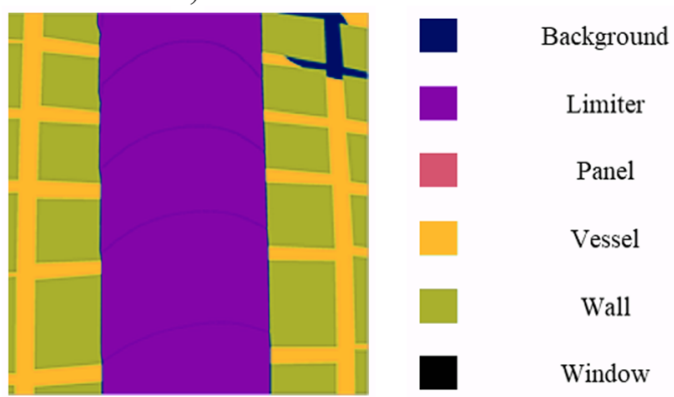

FIG. 6. Projection of the simplified CAD model into the image plane: (a) LWIR camera system and (b) MWIR camera system. the image coordinates $\boldsymbol{p}_{i}$, it is possible to find the camera coordinates $\boldsymbol{c}_{i}$ by inverting with respect to the intrinsic matrix and by undistorting the obtained coordinates. After this, the $3 \mathrm{D}$ coordinates can be evaluated up to a scale factor $d_{i}$ as

$$
\boldsymbol{w}_{i}=d_{i} \boldsymbol{c}_{i} \boldsymbol{R}^{T}-\boldsymbol{t} \boldsymbol{R}^{T} .
$$

The scale factor $d_{i}$ is determined by considering that each line of sight intersects the observed visible surface of the CAD model at only one point, the visible point. Each visible point lies inside a triangle of the observed surface, and all the points belonging to the same triangle lie in a plane surface. Thus, if the CAD model and the line of sight of each pixel are known, it is possible to find the triangle which includes the visible point. The coordinates of the visible point are evaluated by finding the intersection between the triangle plane and the line of sight. After this procedure, the visible point is selected as the point with the minimum scale factor. Figure 6 shows the projection of the full CAD model into the image plane for both LWIR and MWIR camera systems.

\section{B. Surface mapping preserving distances}

Measuring distances on a three-dimensional surface and describing its shape is not a straightforward task since the intrinsic geometry of the surface can significantly differ from the Euclidean geometry. In order to simplify the shape description, a way to project the surface (in our case of the limiter) onto a two-dimensional plane with map projections could be useful. Of course, a side effect of this projection is the occurrence of distortion, given by the fact that three-dimensional surfaces cannot be displayed perfectly in a two-dimensional space. These distortions may depend on which quantities or measures are preserved. Examples of measures which can be distorted are angles, distances, directions, scales, and areas. $^{14}$

In this case, the goal of the map projection is to preserve the distances of the limiter points from two curvilinear axes $u$ and $v$, along the limiter surface in the poloidal and toroidal directions, chosen based on the limiter shape [Fig. 7(a)] and typical heat flux density distribution on the limiter.

To define the position of the strike-line on the limiter, a new spatial orthogonal reference system has been adopted. The origin $\mathrm{O}$ of the new orthogonal coordinate system $X^{\prime} Y^{\prime} Z^{\prime}$ lies in the center of the central tile of the limiter. The $X^{\prime}$ and $Y^{\prime}$ axes lie in the toroidal and poloidal directions, respectively, and $\mathrm{Z}^{\prime}$ is orthogonal to the $X^{\prime} Y^{\prime}$ plane forming a right-handed coordinate system [see Fig. 7(b)]. A front view of the limiter a)

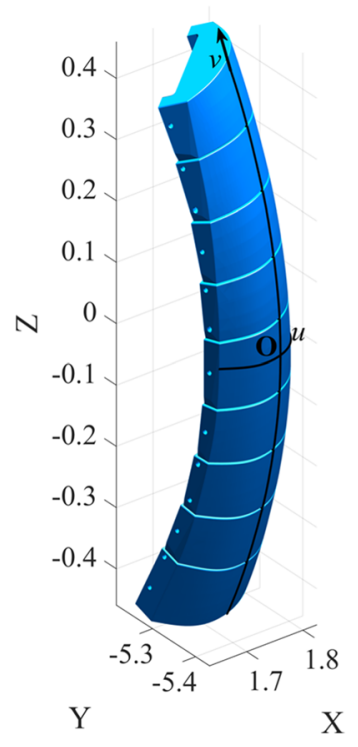

b)

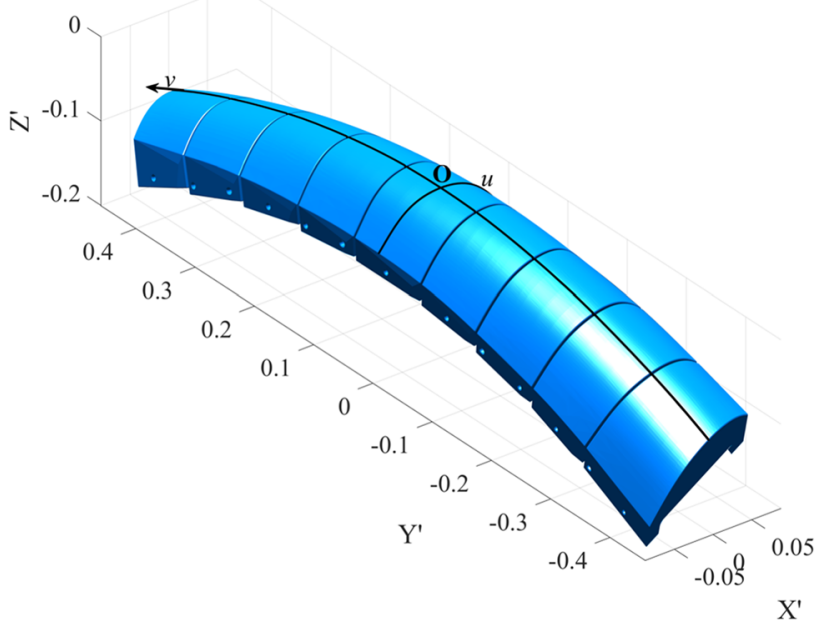

c)

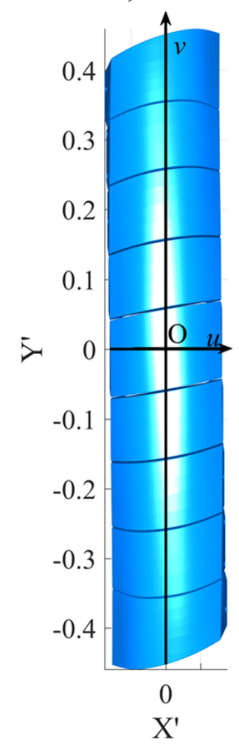

FIG. 7. The limiter on module 5 and reference curvilinear axes: (a) full view in original coordinate system, (b) full view in transformed coordinate system, and (c) front view obtained through a projection on the $X^{\prime} Y^{\prime}$ plane. 
a)

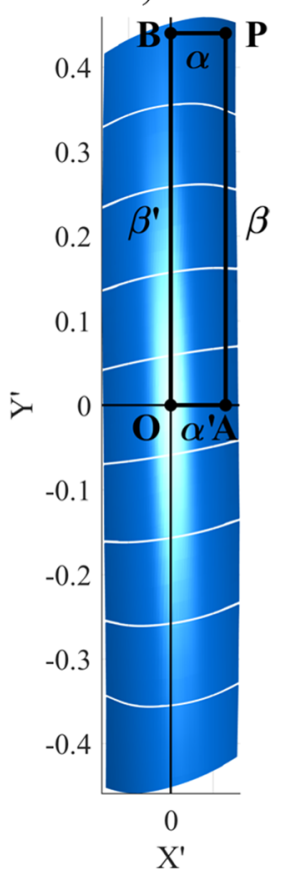

b)

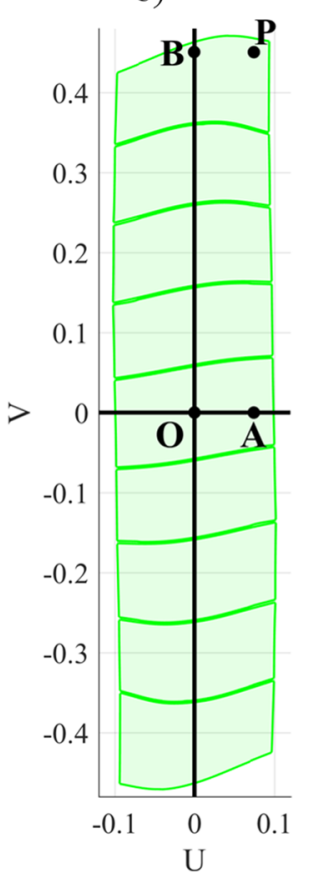

c)

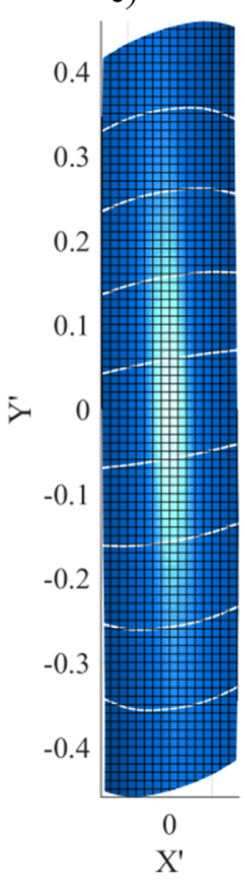

d)

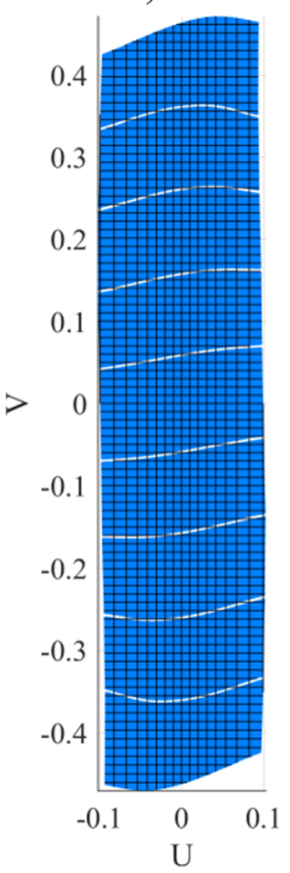

FIG. 8. (a) Limiter model vertices and (b) projection onto the $2 \mathrm{D}$ maps. The contour of the limiter and the projected point $P$ is represented in green for the proposed mapping. (c) Equi-spaced grid on $X^{\prime} Y^{\prime}$ plane and its projection with the (d) proposed mapping. can be seen through an orthogonal projection on the $X^{\prime} Y^{\prime}$ plane [see Fig. 7(c)]. Projecting the points of $X^{\prime}$ and $Y^{\prime}$ axes on the limiter surface, the curvilinear axes $u, v$ are identifiable in the limiter.

Let us now consider a point $\mathrm{P}$ on the $3 \mathrm{D}$ surface of the limiter and two lines parallel to $X^{\prime}$ and $Y^{\prime}$ axes passing through $\mathrm{P}$ [see Fig. 8(a)]. By projecting $X^{\prime}$ and $\mathrm{Y}^{\prime}$ on the limiter surface, two curves $\alpha$ and $\beta$ can be identified in the surface. The curves $\alpha$ and $\beta$ intersect the curvilinear axes $v$ and $u$, respectively, in the points $\mathrm{B}$ and $\mathrm{A}$. Other two curves $\alpha^{\prime}$ and $\beta^{\prime}$, along $u$ and $v$, connect the points $\mathrm{A}$ and $\mathrm{B}$ with the origin $\mathrm{O}$. The arc lengths along the curves $\alpha, \beta, \alpha^{\prime}$ and $\beta^{\prime}$ can be used to find the new coordinates $U, V$ in the new $2 \mathrm{D}$ grid.

In this paper, one mapping is proposed in which points with the same $X^{\prime}\left(Y^{\prime}\right)$ are mapped onto points with the same $U(V)$. Thus, to each point $\mathrm{P}$ on the limiter surface, its $(U, V)$ coordinates can be associated, where

$$
U\left(X^{\prime}\right)=\int_{\alpha^{\prime}} d \alpha^{\prime} V\left(Y^{\prime}\right)=\int_{\beta^{\prime}} d \beta^{\prime}
$$

are arc lengths, equal to the curvilinear distances between points $\mathrm{A}$ and $\mathrm{B}$ and the origin $\mathrm{O}$ along $\alpha^{\prime}$ and $\beta^{\prime}$. The obtained values $U$ and $V$ identify the point $\mathrm{P}$ in the new 2D map.

Through the proposed projection, only curvilinear distances between points belonging to the $U$ axis or to the $V$ axis are preserved. Figure 8(b) shows how the contour of the limiter tiles and the control points $\mathrm{P}, \mathrm{A}$, and $\mathrm{B}$ are projected onto the 2D plane with the proposed mapping. Figures $8(\mathrm{c})$ and 8(d) show how a grid with equidistant knots on $X^{\prime} Y^{\prime}$ plane is distorted with the proposed mapping. As can be noticed, straight lines parallel to $X^{\prime}$ and $Y^{\prime}$ axes remain straight; moreover, since the curvature of the limiter surface has only small variations, the effect of the map distortion may be considered almost negligible.

\section{STRIKE LINE CHARACTERIZATION FROM IR IMAGES}

In order to protect in-vessel components that can be critically damaged by very high heat loads, a real time image processing system is under development at W7-X. This system will provide a rapid signal to the main control system, based on the evaluation of the infrared (IR) measurements. For this reason, a computer-aided analysis of images is a key point to develop tools able to efficiently and automatically process, detect, and analyze the information on the strike-lines. In order to characterize a strike-line, a set of features needs to be defined describing its position, width, and shape. From a spatial point of view, the principal issue is the strike-line segmentation, to be done in the new 2D coordinates system. After the strike-line is segmented, all the strike-line spatial features can be then calculated.

\section{A. Database}

The strike-line characterization process has been implemented analyzing the heat flux density data on the limiter surface. Heat flux data are calculated from the limiter temperature data measured by the infrared cameras. PFCs are, in fact, subject to thermal inertia, and the heat flux is a good measure when estimating the changes of the strike-lines properties during a discharge. The calculation was made for the whole limiter surface and then projected onto the new 2D coordinate system. The calculations of the heat flux density have been made with THEODOR (THermal Energy Onto DivertOR), ${ }^{15}$ a code developed at IPP Garching, by using the spatial calibration results discussed above.

Figures 9 and 10 show the heat flux images and the projections of the heat flux density distribution from both diagnostics (LWIR and MWIR) onto the new 2D coordinate system. The view on Fig. 10 shows a typical distribution of 

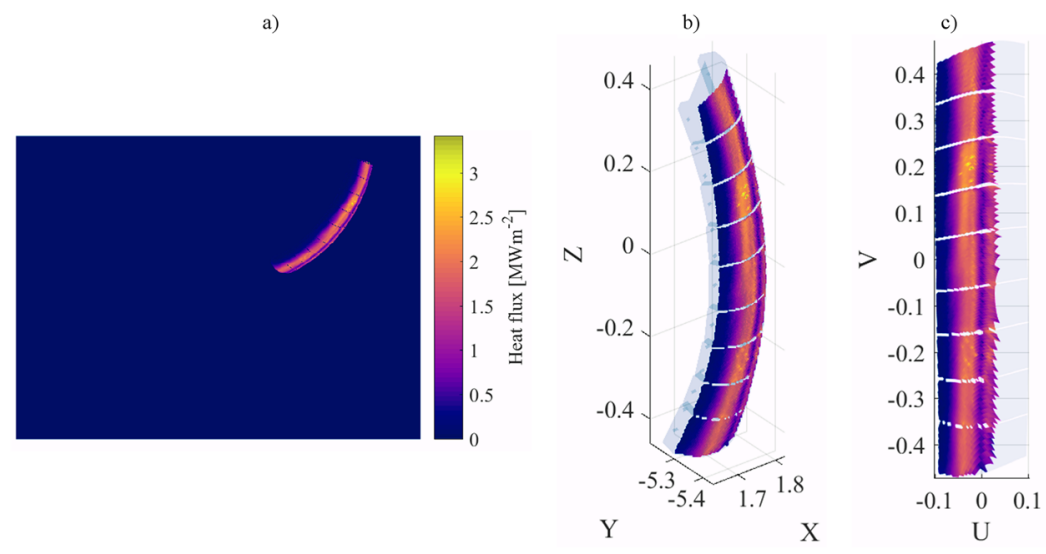

FIG. 9. LWIR camera heat flux image for the limiter of module 5 (a), projection onto the $X Y Z$ reference system (b), and projection onto the 2D coordinate system (c).

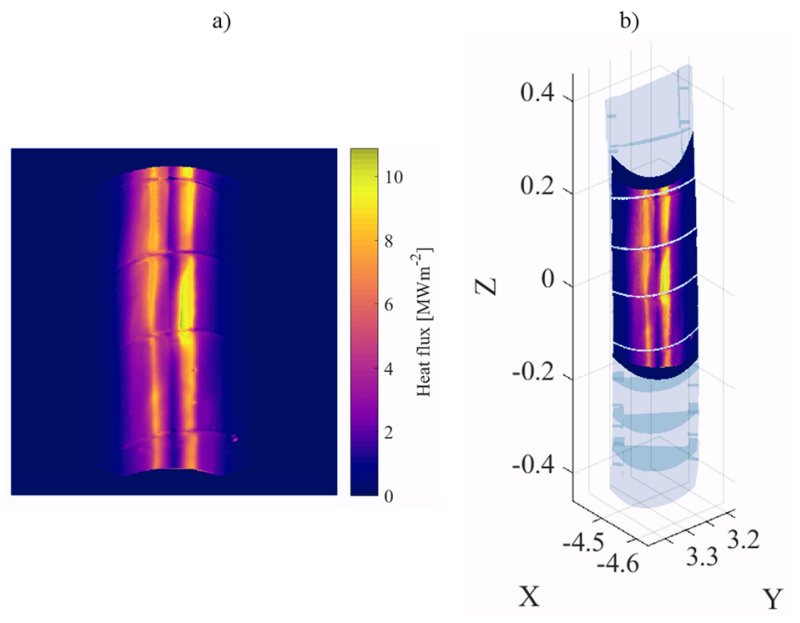

the power loads for OP1.1 with two strike lines aligned along the vertical axis of the limiter. A comparison of Figs. 9 and 10 shows that the MWIR camera provides partial images of both limiter strike lines at higher spatial resolution, but the LWIR camera provides the image of the whole strikeline on the left part of the limiter surface. Therefore, further analyses are made based on the data from the LWIR camera.

A dataset of 12 experiments with different strike-line shapes has been used in order to test the characterization methodology. This dataset is characterized by different sets of experimental parameters, including ECRH (Electron

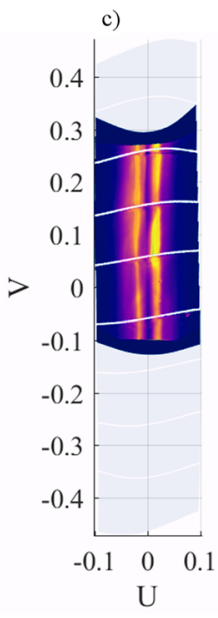

FIG. 10. MWIR camera heat flux image for the limiter of module 3 (a), projection onto the $X Y Z$ reference system (b), and projection onto the $2 \mathrm{D}$ coordinate system (c).

TABLE I. Experimental conditions for the different experiments.

\begin{tabular}{|c|c|c|c|c|c|c|c|c|c|}
\hline \# & $\mathrm{I}_{1}(\mathrm{~A})$ & $\mathrm{I}_{2}(\mathrm{~A})$ & $\mathrm{I}_{3}(\mathrm{~A})$ & $\mathrm{I}_{4}(\mathrm{~A})$ & $\mathrm{I}_{5}(\mathrm{~A})$ & $\mathrm{I}_{\mathrm{A}}(\mathrm{A})$ & $\mathrm{I}_{\mathrm{B}}(\mathrm{A})$ & $\mathrm{P}_{\mathrm{ECRH}}(\mathrm{MW})$ & $\mathrm{n}_{\mathrm{e}}\left(10^{20} \mathrm{~m}^{-2}\right)$ \\
\hline 1 & 12362 & 12364 & 12362 & 12363 & 12371 & 4817 & 4826 & 2 & 0.25 \\
\hline 2 & 12362 & 12364 & 12362 & 12363 & 12371 & 4817 & 4826 & 4 & 0.4 \\
\hline 3 & 12362 & 12364 & 12362 & 12363 & 12371 & 4817 & 4826 & 0.5 & 0.1 \\
\hline 4 & 12362 & 12364 & 12362 & 12363 & 12371 & 4817 & 4826 & 2 & 0.25 \\
\hline 5 & 12362 & 12363 & 12362 & 12362 & 12371 & 4817 & 4826 & 2.5 & 0.2 \\
\hline 6 & 12608 & 12610 & 12608 & 12610 & 12618 & 4913 & 4922 & 2.5 & 0.25 \\
\hline 7 & 12370 & 12372 & 12370 & 12371 & 12379 & 4821 & 4025 & 0.6 & 0.1 \\
\hline 8 & 12379 & 12380 & 12379 & 12379 & 12388 & 4821 & 3223 & 2 & 0.25 \\
\hline 9 & 12387 & 12388 & 12387 & 12387 & 12396 & 4827 & 2420 & 2 & 0.25 \\
\hline 10 & 12412 & 12414 & 12413 & 12412 & 12421 & 4834 & 5 & 2 & 0.25 \\
\hline 11 & 12412 & 12413 & 12413 & 12412 & 12421 & 4834 & 5 & 2 & 0.25 \\
\hline 12 & 12412 & 12413 & 12413 & 12412 & 12421 & 4834 & 5 & 2.5 & 0.2 \\
\hline
\end{tabular}



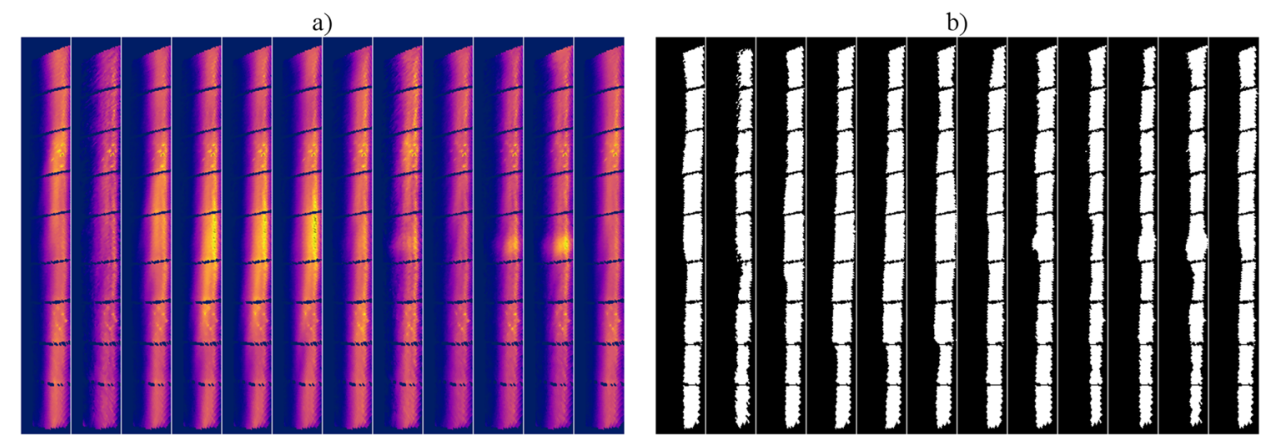

FIG. 11. Left strike-line images (a) projected in the $2 \mathrm{D}$ grid for the 12 experiments and (b) segmented.
$2 \mathrm{~mm} /$ pixel [see Fig. 11(a)]. The center of the new image corresponds to the origin of the $2 \mathrm{D}$ grid. The following criteria were used to segment the measured strike-lines: if a point in the 2D coordinate system has a value of either $U<0$ or $U>0$, then it belongs either to the left-hand side or right-hand side strikeline. When the two strike-lines are separated into two different images, each strike-line image therefore consists of $60 \times 481$ pixels.

In order to select the valid frames to be analyzed, a lower limit on the maximum limiter heat flux $\phi_{\max }$ in each frame has been set: if $\phi_{\max }$ is greater than or equal to $\Phi_{\max }$ $\exp (-1)$, where $\Phi_{\max }$ is the maximum limiter heat flux during the entire discharge, then the frame is considered as valid. With this selection, from a total of 820 frames in the dataset, 489 valid frames with a strike-line have been selected. In each valid frame, the strike line region has been identified by considering those pixels, which (a) lie within the limiter region and (b) show heat flux density greater than or equal to $\phi_{\text {th }}=$ $\phi_{\max } \exp (-1)$. In Fig. 11, the left strike-line at the steady state is shown for the 12 experiments before and after the segmentation.

From the segmented strike-line image, the horizontal position was calculated as the mean value of the horizontal coordinate $(\mathrm{U})$ of the strike-line edge points inside the 2D grid.
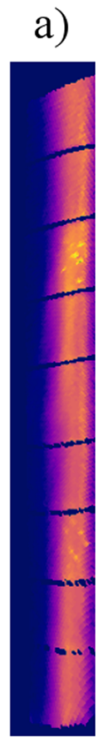

b)

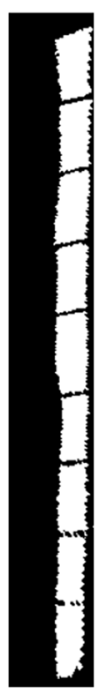

c)

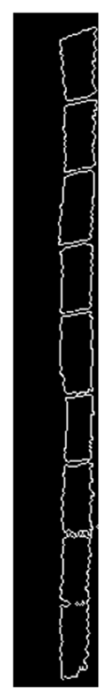

d)

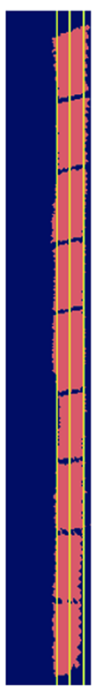

FIG. 12. Image obtained by projecting the left strike-line in the 2D grid, and the process to estimate its horizontal position and width. (a) Left strike line projected, (b) segmented strike-line shape, (c) edge of the strike-line, and (d) estimated position and width.
The strike-line width was calculated as two times the standard deviation of the horizontal coordinate of the same set of edge points. Figure 12 shows the entire process to estimate horizontal position and width of the strike-line starting from the projected image. Figure 13 shows the evolution of the strike-line horizontal position and width during the first experiment. As can be noticed, at the beginning and at the end of the experiment, the strike-line region is heavily fragmented, due to the fact that the discharge has not reached the steady-state yet.

\section{Strike-line shape descriptors}

Shape descriptors can be classified into two main classes: contour-based, which exploit only shape boundary information, and region-based, in which all the pixels within the shape region are used to estimate the features. For each class, global and structural approaches can be distinguished, based on whether the shape is represented as a whole or by its subparts. ${ }^{16}$

The necessity to describe the strike-line shape with a limited and standard number of features, also in the case of disjoint shapes, led to the choice of global region-based methods. These methods in fact treat a shape, no matter if disjoint or not, as a whole, representing it as a feature vector. In this way, similarity among the shapes of different strike-lines can be measured by estimating the distance between their feature vectors.

The quality of a shape descriptor is measured by its effectiveness and efficiency to find perceptually similar shapes in a database. Usually it means to find features, which are invariant with respect to rotation, translation, and scaling. In our particular case, rotation, translation, and scaling of the strikeline are themselves important features and have been taken into account to distinguish the strike-lines. A 2D FFT has been thus applied to the segmented strike-line image [Fig. 11(b)] in order to obtain the Fourier Descriptors. The Fourier Descriptors have been kept without normalization in order to preserve the sensitivity to scale.

As expected, the majority of the FFT points have a negligible magnitude, and the most relevant points are positioned in the low frequency regions of the spectrum. Moreover, due to the fact that the amplitude of Fourier coefficients is symmetrical with respect to the anti-diagonal, only roughly one half of them needs to be stored. This set of Fourier coefficients is the set of descriptors which are used to define the strike line shape. 
a)

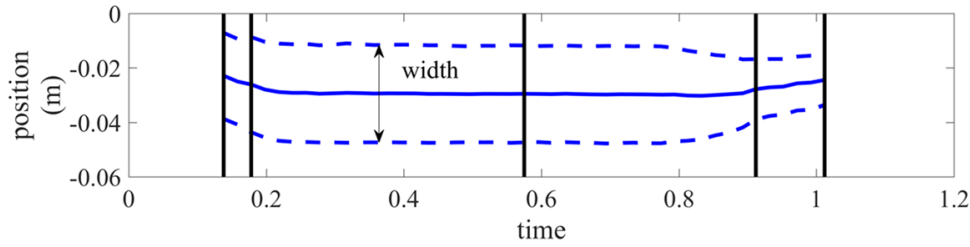

$(\mathrm{s})$
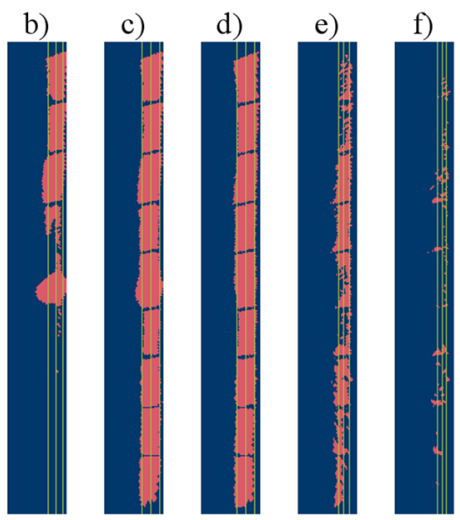

FIG. 13. (a) Evolution of strike-line position and width during experiment \#1 and (b)-(f) related segmented frames [the time of each frame is given by the black lines in (a)].

This set of descriptors can be used in order to reconstruct the strike-line shape by means of an inverse FFT followed by a binarization. A study has been made in order to find a reduced set of descriptors able to provide a good reconstruction of the strike-line shape, in the case of both fragmented and full strikelines. To this end, for each frame, the descriptors magnitude has been calculated and normalized with respect to the maximum value in the frame. Then, the coefficient indices have been ranked with respect to their maximum normalized magnitude among all frames, in this way defining the relevance of each coefficient.

A dilated version ${ }^{17}$ of the original segmented image, in which the shape boundary radius was increased by one pixel, has been used as a measure of tolerance in the reconstruction error and as a reference of goodness. The reconstruction was considered as successful if the error for the reconstructed image $\varepsilon_{\text {rec }}$, computed as the percentage of wrong pixel values, was equal to or lower than the error related to the dilated image $\varepsilon_{d i l}$. The error $\varepsilon_{d i l}$ has been used as a reference because a small and tolerable error in the boundary could lead to high errors (in terms of percentage of wrong pixels)

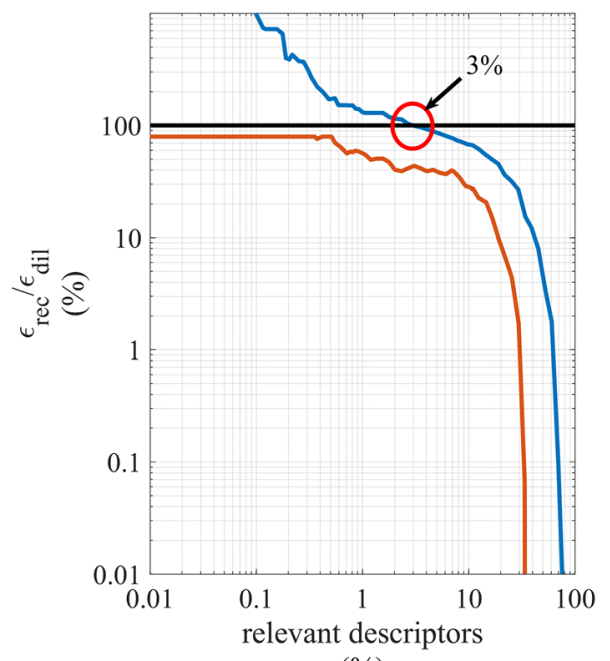

$(\%)$

FIG. 14. Maximum (blue) and minimum (red) reconstruction error divided by the tolerated error, in percentage, depending on the percentage of descriptors used for the reconstruction. in the case of fragmented objects, when the ratio between the perimeter and the area is large.

Figure 14 shows the maximum and minimum ratio $\varepsilon_{\text {rec }} / \varepsilon_{\text {dil }}$, versus the percentage of relevant descriptors. As can be seen from the maximum reconstruction error, in order to reach a tolerable reconstruction error for all frames, at least the $3 \%$ of most relevant descriptors is necessary. It is worth noting that there are frames for which the reconstruction error is acceptable with a smaller number of descriptors: this is the case of steady-state strike-line images, in which the object is less fragmented and simpler to describe. On the contrary, when the strike-line is fragmented, e.g., at the beginning or at the ending of the experiment, a higher number of descriptors are needed, leading to a percentage of relevant descriptors, necessary for a proper reconstruction, equal to $3 \%$.

Figure 15 shows the goodness of reconstruction of the strike-line shape for two cases, by using $100 \%, 20 \%, 3 \%$, $0.5 \%$, and $0.01 \%$ of descriptors. As can be noticed, in the first example, the reconstruction is good also with a percentage of coefficients lower than 3\%. This is not the case of the second example, in which with a percentage of $0.01 \%$ of descriptors, the strike-line cannot be reconstructed at all. a)

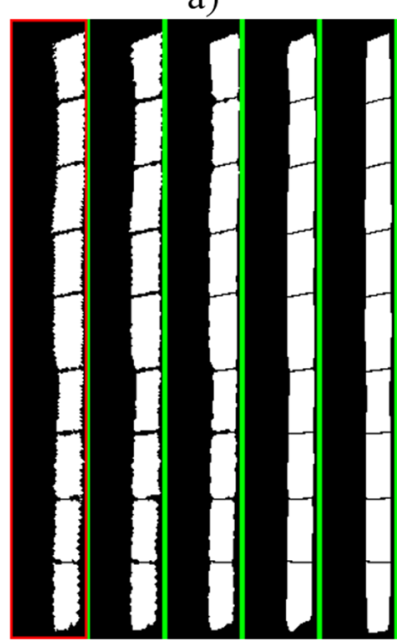

b)

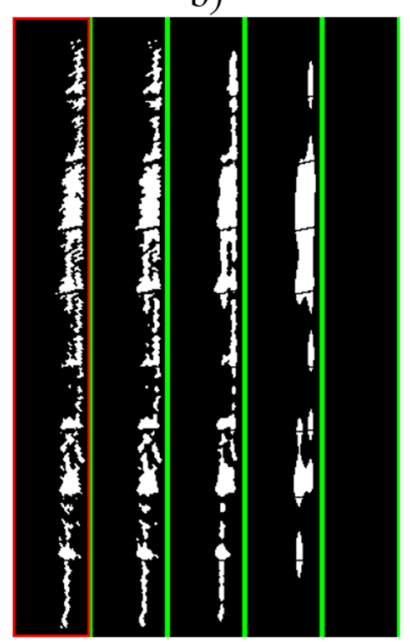

FIG. 15. Strike-line shape reconstruction for two examples [(a) and (b)] by using (from left to right) $100 \%, 20 \%, 3 \%, 0.5 \%$, and $0.01 \%$ of the most relevant descriptors. 


\section{CONCLUSIONS}

In this work, a new image processing system for the analysis of thermal events from the first W7-X experimental campaign has been presented. The analysis of thermal events has taken into account the real position of PFCs by means of a spatial calibration of the IR cameras and a simplified CAD model. This method has allowed projecting the thermal events from IR images on the 3D surface of a CAD model of the limiter and then on a specific 2D grid. The analysis of thermal events on the limiter has been then made in terms of spatial features, describing the strike-line position, width, and shape.

The proposed image processing system will be updated and applied to the analysis of thermal events during the next experimental campaigns. The more complex structure of the divertor surfaces will add some more issues on the definition of the strike-lines. Thus, the strike-line descriptors, in particular the strike-line position, must be re-defined with respect to the divertor shape.

Further work is needed to apply the image processing algorithms to real-time operations. In this context, the system is a step towards the development of the near real-time image diagnostic system in $\mathrm{W} 7-\mathrm{X}^{18}$ for the protection of the PFCs against damages due to local overheating during plasma operations.

\section{ACKNOWLEDGMENTS}

This work has been carried out within the framework of the EUROfusion Consortium and has received funding from the Euratom research and training programme 2014-2018 and 2019-2020 under Grant Agreement No. 633053. The views and opinions expressed herein do not necessarily reflect those of the European Commission.

${ }^{1}$ B. Sieglin, M. Faitsch, A. Herrmann, B. Brucker, T. Eich, L. Kammerloher, and S. Martinov, "Real time capable infrared thermography for ASDEX upgrade," Rev. Sci. Instrum. 86, 113502 (2015).

${ }^{2}$ M. W. Jakubowski, P. Drewelow, J. Fellinger, A. Puig Sitjes, G. Wurden, A. Ali, C. Biedermann, B. Cannas, D. Chauvin, M. Gamradt, H. Greve, Y. Gao, R. Hathiramani, R. König, A. Lorenz, V. Moncada, H. Niemann, T. T. Ngo, F. Pisano, T. Sunn Pedersen, and W7-X Team, "Infrared imaging systems for wall protection in the W7-X stellarator," Rev. Sci. Instrum. 89, 10E116 (2018).
${ }^{3}$ R. C. Wolf et al., "Major results from the first plasma campaign of the Wendelstein 7-X stellarator," Nucl. Fusion 57(10), 102020 (2017).

${ }^{4}$ M. Krychowiak et al., "Overview of diagnostic performance and results for the first operation phase in Wendelstein 7-X," Rev. Sci. Instrum. 87, 11D304 (2016).

${ }^{5}$ D. Wassilew, U. Hoffmann, and D. Hildebrandt, "Development of an IR microbolometer camera for operation in a strong magnetic field," in Proceedings of the 12th International Conference on Infrared Sensors and Systems, Nürnberg (AMA Association for Sensors and Measurement, 2011), pp. 115-118.

${ }^{6} \mathrm{R}$. König et.al., "Diagnostics design for steady-state operation of the Wendelstein 7-X stellarator," Rev. Sci. Instrum. 81, 10E133 (2010).

${ }^{7} \mathrm{R}$. König et al., "The set of diagnostics for the first operation campaign of the Wendelstein 7-X stellarator," J. Instrum. 10, P10002 (2015).

${ }^{8}$ H. Niemann, M. W. Jakubowski, T. Sunn Pedersen, R. König, G. A. Wurden, F. Effenberg, D. Zhang, and W7-X Team, "Power loads in the limiter phase of Wendelstein 7-X," in 43rd EPS Conference on Plasma Physics, Geneva, edited by P. Mantica (European Physical Society, 2016).

${ }^{9}$ G. A. Wurden, L. A. Stephey, C. Biedermann, M. W. Jakubowski, J. P. Dunn, M. Gamradt, and W7-X Team, "A high resolution IR/visible imaging system for the W7-X limiter," Rev. Sci. Instrum. 87, 11 D607 (2016).

${ }^{10}$ G. A. Wurden, C. Biedermann, F. Effenberg, M. Jakubowski, H. Niemann, L. Stephey, S. Bozhenkov, S. Brezinsek, J. Fellinger, B. Cannas, F. Pisano, S. Marsen, H. P. Laqua, R. König, O. Schmitz, J. H. Harris, E. A. Unterberg, and W7-X Team, "Limiter observations during W7-X first plasmas," Nucl. Fusion 57, 056036 (2017).

${ }^{11}$ D. A. Forsyth and J. Ponce, Computer Vision, a Modern Approach (Prentice Hall, 2003).

${ }^{12}$ P. Sturm, "Pinhole camera model," in Computer Vision, edited by K. Ikeuchi (Springer Science+Business Media, New York, 2016), Vol. 2014, pp. 610613.

${ }^{13}$ Z. Zhang, "A flexible new technique for camera calibration," IEEE Trans. Pattern Anal. Mach. Intell. 22(11), 1330-1334 (2000).

${ }^{14}$ J. P. Snyder, Flattening the Earth: Two Thousand Years of Map Projections (University of Chicago Press, 1993).

${ }^{15}$ A. Herrmann and ASDEX Upgrade Team, "Limitations for divertor heat flux calculations of fast events in tokamaks," in 28th EPS Conference on Controlled Fusion and Plasma Physics, Geneva, edited by C. Silva, C. Varandas, and D. Campbell (European Physical Society, 2001), pp. 2109-2112, Contributed Paper.

${ }^{16} \mathrm{D}$. Zhang and G. Lu, "Review of shape representation and description techniques," Pattern Recognit. 37(1), 1-19 (2004).

${ }^{17}$ R. C. Gonzalez and R. W. Woods, Digital Image Processing (Prentice Hall, 2002).

${ }^{18}$ A. Puig Sitjes, M. Jakubowski, A. Ali, P. Drewelow, V. Moncada, F. Pisano, T. T. Ngo, B. Cannas, J. M. Travere, G. Kocsis, T. Szepesi, T. Szabolics, and W7-X Team, Wendelstein 7-X Near Real-Time Image Diagnostic System for Plasma-Facing Components Protection, Fusion Science and Technology (Taylor \& Francis, 2017). 